\title{
Quantification of pulmonary perfusion abnormalities using DCE-MRI in COPD: comparison with quantitative $\mathrm{CT}$ and pulmonary function
}

\author{
Marilisa Schiwek $^{1,2,3} \cdot$ Simon M. F. Triphan ${ }^{1,3} \cdot$ Jürgen Biederer ${ }^{1,3,4,5} \cdot$ Oliver Weinheimer $^{1,3} \cdot$ Monika Eichinger $^{1,3,6}$. \\ Claus F. Vogelmeier ${ }^{7} \cdot$ Rudolf A. Jörres $^{8} \cdot$ Hans-Ulrich Kauczor ${ }^{1,3} \cdot$ Claus P. Heußel $^{1,3,6}$. Philip Konietzke ${ }^{1,3}$. \\ Oyunbileg von Stackelberg ${ }^{1,3} \cdot$ Frank Risse $^{2} \cdot$ Bertram J. Jobst $^{1,3} \cdot$ Mark O. Wielpütz $^{1,3}$. . on behalf of the \\ COSYCONET study group
}

Received: 7 May 2021 / Revised: 29 June 2021 / Accepted: 26 July 2021 / Published online: 22 September 2021

c) The Author(s) 2021, corrected publication 2022

\begin{abstract}
Objectives Pulmonary perfusion abnormalities are prevalent in patients with chronic obstructive pulmonary disease (COPD), are potentially reversible, and may be associated with emphysema development. Therefore, we aimed to evaluate the clinical meaningfulness of perfusion defects in percent (QDP) using DCE-MRI.

Methods We investigated a subset of baseline DCE-MRIs, paired inspiratory/expiratory CTs, and pulmonary function testing (PFT) of 83 subjects (age $=65.7 \pm 9.0$ years, patients-at-risk, and all GOLD groups) from one center of the "COSYCONET" COPD cohort. QDP was computed from DCE-MRI using an in-house developed quantification pipeline, including four different approaches: Otsu's method, k-means clustering, texture analysis, and $80^{\text {th }}$ percentile threshold. QDP was compared with visual MRI perfusion scoring, CT parametric response mapping $(\mathrm{PRM})$ indices of emphysema $\left(\mathrm{PRM}_{\mathrm{Emph}}\right)$ and functional small airway disease $\left(\mathrm{PRM}_{\mathrm{fSAD}}\right)$, and FEV1/FVC from PFT. Results All QDP approaches showed high correlations with the MRI perfusion score $(r=0.67$ to $0.72, p<0.001)$, with the highest association based on Otsu's method $(r=0.72, p<0.001)$. QDP correlated significantly with all PRM indices $(p<0.001)$, with the strongest correlations with $\mathrm{PRM}_{\mathrm{Emph}}(r=0.70$ to $0.75, p<0.001)$. QDP was distinctly higher than PRM $\mathrm{Emph}$ (mean difference $=35.85$ to 40.40 ) and $\mathrm{PRM}_{\mathrm{fSAD}}$ (mean difference $=15.12$ to 19.68), but in close agreement when combining both PRM indices (mean difference $=1.47$ to 6.03) for all QDP approaches. QDP correlated moderately with FEV1/FVC $(r=-0.54$ to $-0.41, p<0.001)$. Conclusion QDP is associated with established markers of disease severity and the extent corresponds to the CT-derived combined extent of PRM $\mathrm{Emph}_{\text {and PRM }}$ fSAD. We propose to use QDP based on Otsu's method for future clinical studies in COPD. Key Points

- QDP quantified from DCE-MRI is associated with visual MRI perfusion score, CT PRM indices, and PFT.

- The extent of QDP from DCE-MRI corresponds to the combined extent of PRM $M_{E m p h}$ and PRM fSAD from CT.

- Assessing pulmonary perfusion abnormalities using DCE-MRI with QDP improved the correlations with CT PRM indices and PFT compared to the quantification of pulmonary blood flow and volume.
\end{abstract}

Mark O. Wielpütz

Mark.Wielpuetz@med.uni-heidelberg.de

1 Department of Diagnostic and Interventional Radiology, Subdivision of Pulmonary Imaging, University Hospital of Heidelberg, Im Neuenheimer Feld 420, 69120 Heidelberg, Germany

2 Boehringer Ingelheim Pharma GmbH \& Co. KG, Birkendorfer Strasse 65, 88397 Biberach an der Riß, Germany

3 Translational Lung Research Center Heidelberg (TLRC), German Lung Research Center (DZL), Im Neuenheimer Feld 156, 69120 Heidelberg, Germany

4 Faculty of Medicine, University of Latvia, Raina bulvaris 19, Riga 1586, Latvia
5 Faculty of Medicine, Christian-Albrechts-Universität Zu Kiel, 24098 Kiel, Germany

6 Department of Diagnostic and Interventional Radiology with Nuclear Medicine, Thoraxklinik at the University Hospital of Heidelberg, Röntgenstr. 1, 69126 Heidelberg, Germany

7 Department of Medicine, Pulmonary and Critical Care Medicine, Philipps-University of Marburg (UMR), Marburg, Germany

8 Institute and Outpatient Clinic for Occupational, Social and Environmental Medicine, University Hospital, Ludwig Maximilians University (LMU) Munich, Comprehensive Pneumology Center Munich (CPC-M), Munich, Germany 
Keywords Perfusion imaging $\cdot$ Biomarkers $\cdot$ Magnetic resonance imaging $\cdot$ Chronic obstructive pulmonary disease .

Pulmonary emphysema

$\begin{array}{ll}\text { Abbreviations } & \\ \text { AIF } & \text { Arterial input function } \\ \text { ANOVA } & \text { One-way analysis of variance } \\ \text { CA } & \text { Contrast agent } \\ \text { CCA } & \text { Cross-correlation analysis } \\ \text { COPD } & \text { Chronic obstructive pulmonary disease } \\ \text { DCE-MRI } & \begin{array}{l}\text { Dynamic contrast-enhanced magnetic } \\ \text { resonance imaging }\end{array} \\ \text { FD } & \text { Fourier decomposition } \\ \text { FEV1\%predicted } & \text { Forced expiratory volume in 1 s per- } \\ & \text { cent predicted } \\ \text { FEV1/FVC } & \text { Ratio between forced expiratory vol- } \\ & \text { ume in 1 s and forced vital capacity } \\ \text { fSAD } & \text { Functional small airways disease } \\ \text { HPV } & \text { Hypoxic pulmonary vasoconstriction } \\ \text { PBF } & \text { Pulmonary blood flow } \\ \text { PBV } & \text { Pulmonary blood volume } \\ \text { PFT } & \text { Pulmonary function testing } \\ \text { PRM } & \text { Parametric response mapping } \\ \text { QDP } & \text { Perfusion defects in percent } \\ \text { Rmax map } & \text { Residue function map at the time point } \\ & \text { of maximum contrast enhancement } \\ \text { R(t) map } & \text { Time-resolved residue function map }\end{array}$

\section{Introduction}

COPD is characterized by progressive airflow limitation caused by airway obstruction and emphysematous lung destruction. The resulting regional alveolar hypoxia leads to hypoxic pulmonary vasoconstriction (HPV), reducing pulmonary perfusion regionally. Moreover, the alveolar-capillary bed and pulmonary vessels are obliterated by emphysematous destruction. Both processes, HPV and the loss of lung capillaries, become apparent on functional imaging as regional perfusion abnormalities. DCE-MRI is an established technique to assess regional perfusion abnormalities by exploiting the contrast enhancement in lung parenchyma during the first pass of an i.v. injected contrast agent (CA) bolus. In clinical studies, perfusion abnormalities using DCE-MRI are assessed either by visual scoring systems or by computational methods for quantitative evaluation [ 1 , 2]. The use of semi-quantitative scoring systems to monitor treatment effects on pulmonary perfusion might be challenging in early clinical studies designed to test treatments that slow emphysema progression. Due to the relatively slow disease progression in COPD and the short follow-up-intervals (up to 3 months) in early clinical studies, only relatively subtle changes in pulmonary perfusion are expected in such settings, which may remain undetected by scoring systems [3].
In previous studies, pulmonary blood flow (PBF) and pulmonary blood volume (PBV) were used to quantify pulmonary perfusion from DCE-MRI $[1,4,5]$. Particularly interesting for clinical studies, the quantification by computer algorithms limits human interaction and generates potentially more objective results than visual assessments. However, DCE-MRI in the lung is adversely affected by low contrast-to-noise ratios, non-linearities of the CA-signal relationship [6, 7], and pronounced image artefacts, which impair the reproducibility and robustness of PBF and PBV $[8,9]$. The introduction of unsupervised image clustering algorithms on Fourier decomposition (FD), hyperpolarized helium, and xenon MRI enabled the quantification of the extent of perfusion or ventilation abnormalities relative to the lung volume ("defect-percent") [10-13]. It has been shown that "defect-percent" is a sensitive marker for disease severity based on the observed correlations with spirometry, multiple breath washout, and morphological abnormalities [14-16]. However, different "defect-percent" quantification approaches were used and studies using DCE-MRI or their application in sizeable datasets are mostly missing.

The aims of this work were to (a) develop a robust algorithm to quantify QDP using DCE-MRI by comparing different approaches and (b) investigate the clinical meaningfulness of QDP by comparing it with a visual MRI perfusion score, quantitative CT PRM indices of emphysema, and functional small airways disease (fSAD), and PFT. Furthermore, we compared the performance of QDP with that of PBF and PBV.

\section{Materials and methods}

\section{Study design and study population}

The study is based on data from a prospective longitudinal multicenter imaging sub-study of the COPD cohort "Impact of Systemic Manifestations/Comorbidities on Clinical State, Prognosis, Utilisation of Health Care Resources in Patients with COPD"-trial (COSYCONET (NCT01245933); substudy "Image-Based Structural and Functional Phenotyping of the COSYCONET Cohort Using MRI and CT" (MR-COPD, NCT02629432)). The inclusion and exclusion criteria of COSYCONET can be found elsewhere [17]. In addition to subjects of the GOLD 1-4 categories [18], smokers and former smokers with no assignable GOLD category including the former GOLD 0 and subjects at risk for COPD were enrolled. The "former GOLD 0" group includes subjects with normal PFT in terms of FEV1/FVC (ratio between forced expiratory volume in $1 \mathrm{~s}$ and forced vital capacity) but with COPD-specific symptoms and the "at risk for COPD" group includes 
subjects not classifiable within GOLD, with normal PFT in terms of FEV1/FVC and without COPD-specific symptoms. Institutional ethics committee approval was obtained, and all subjects gave their written informed consent.

For this work, a subset of 103 subjects examined at the same center was selected and only baseline data were used.

\section{MRI acquisition}

All subjects underwent MRI using the same $1.5 \mathrm{~T}$ scanner (Magnetom Aera, Siemens Healthineers) using a standardized chest MRI protocol (Supplementary table 1) [3, 19, 20], validated by a phantom study [21]. For the DCE-MRI perfusion imaging, a time-resolved T1-weighted 3D keyhole pulse sequence (time-resolved angiography with interleaved stochastic trajectories [TWIST]) with a fixed dose of $2 \mathrm{ml}$ gadolinium-based CA (Gadobutrol, Bayer Vital GmbH) was injected i.v. at $4 \mathrm{ml} / \mathrm{s}$ followed by a saline chaser [22, 23]. The DCE-MRI ( $33 \mathrm{~s}$, temporal resolution $\sim 1.6 \mathrm{~s})$ was acquired in inspiratory breath-hold.

\section{MRI perfusion score}

Visual scoring of DCE-MRI was performed by an experienced radiologist using a validated MRI perfusion score $[2,3,20,24]$. The extent of perfusion abnormalities was assessed on lobe level as follows: $0=$ no abnormality, $1=<50 \%$ of the lobe involved, and $2=\geq 50 \%$ of the lobe involved and summed for the whole lung, resulting in a maximum range between zero and twelve.

\section{MRI quantification pipeline}

The in-house developed MRI analysis pipeline was written in MATLAB (R2019a, The MathWorks, Inc.). All image process steps were performed fully automatically unless otherwise specified. Further details are provided in the Supplementary materials.

\section{Image pre-processing}

Time-resolved subtraction images of the DCE-MRI were generated by subtracting the mean of the two first pre-contrast images. Arterial input functions (AIFs) were calculated in the pulmonary artery [25]. Time-resolved residue function maps $(\mathrm{R}(\mathrm{t})$ map) were computed by deconvolving voxel-by-voxel the AIF with each voxel of the subtraction image using truncated singular value decomposition [26]. The model used for the $\mathrm{R}(\mathrm{t})$ map calculation is based on the principles of tracer kinetics for non-diffusible tracers [27, 28]. Minor respiratory motion artefacts, usually occurring near the diaphragm, were excluded using cross-correlation analysis.

\section{Quantification of pulmonary perfusion abnormalities}

The lungs were automatically segmented from coronal T1-weighted images [29], registered to DCE-MRI images, and reviewed individually by an investigator. For comparison with the visual perfusion score, an approximate division of each lung into lobes was performed (Supplementary Fig. 1).

Pulmonary perfusion abnormalities in DCE-MRI are characterized by absent or delayed contrast enhancement. QDP was quantified from $\mathrm{R}(\mathrm{t})$ maps at the time point of maximum contrast enhancement $\left(R_{\max }\right.$ map) using four different approaches based on unsupervised image clustering algorithms: Otsu's method, k-means clustering, texture analysis and percentilethreshold. It was calculated in percent representing the extent of perfusion abnormalities relative to the segmented lung volume, with a theoretical range between 0 and $100 \%$. Details about the four different calculation approaches are outlined in Fig. 1 and in the Supplementary materials.

For the comparison between QDP and the MRI perfusion score by lobe, QDP was converted into discrete values at lobe level (Supplementary table 2) [30]. The quantification of the pulmonary perfusion metrics PBF and PBV was based on the $\mathrm{R}(\mathrm{t})$ map as described elsewhere [4].

\section{CT parametric response mapping}

All subjects underwent standardized phantom-controlled (Catphan600, The Phantom Laboratory) same-day nonenhanced low-dose CT (Somatom 64, Siemens Healthineers) with paired scans in inspiratory and expiratory breath-hold at $120 \mathrm{kV}$ and $35 \mathrm{mAs}$. Images were reconstructed at $1.0 \mathrm{~mm}$ slice thickness with a $0.5-\mathrm{mm}$ interval using a soft filtered back-projection convolution kernel (B30f). CT scans were post-processed using the in-house software YACTA as described previously (version 2.8.7) [31, 32]. PRM classified the lung in normal $\left(\mathrm{PRM}_{\text {Normal }}\right)$, emphysematous $\left(\mathrm{PRM}_{\mathrm{Emph}}\right)$, or fSAD (PRM $\left.{ }_{\mathrm{fSAD}}\right)$ [33]. PRM was calculated in percent relative to the segmented lung volume. $\mathrm{PRM}_{\mathrm{Abnormal}}$ was computed to describe the proportion of non-normal lung tissue as $\mathrm{PRM}_{\text {Abnormal }}=\mathrm{PRM}_{\mathrm{Emph}}+\mathrm{PRM}_{\mathrm{fSAD}}$.

\section{Pulmonary function testing}

PFT was performed according to the American Thoracic Society and European Respiratory Society recommendations [34]. In this study, forced expiratory volume in $1 \mathrm{~s}$ percent predicted (FEV1\% predicted) and FEV1/FVC were used.

\section{Statistical analysis}

Statistical analyses were performed using R (R 3.3.2, Foundation for Statistical Computing). Data are presented as 


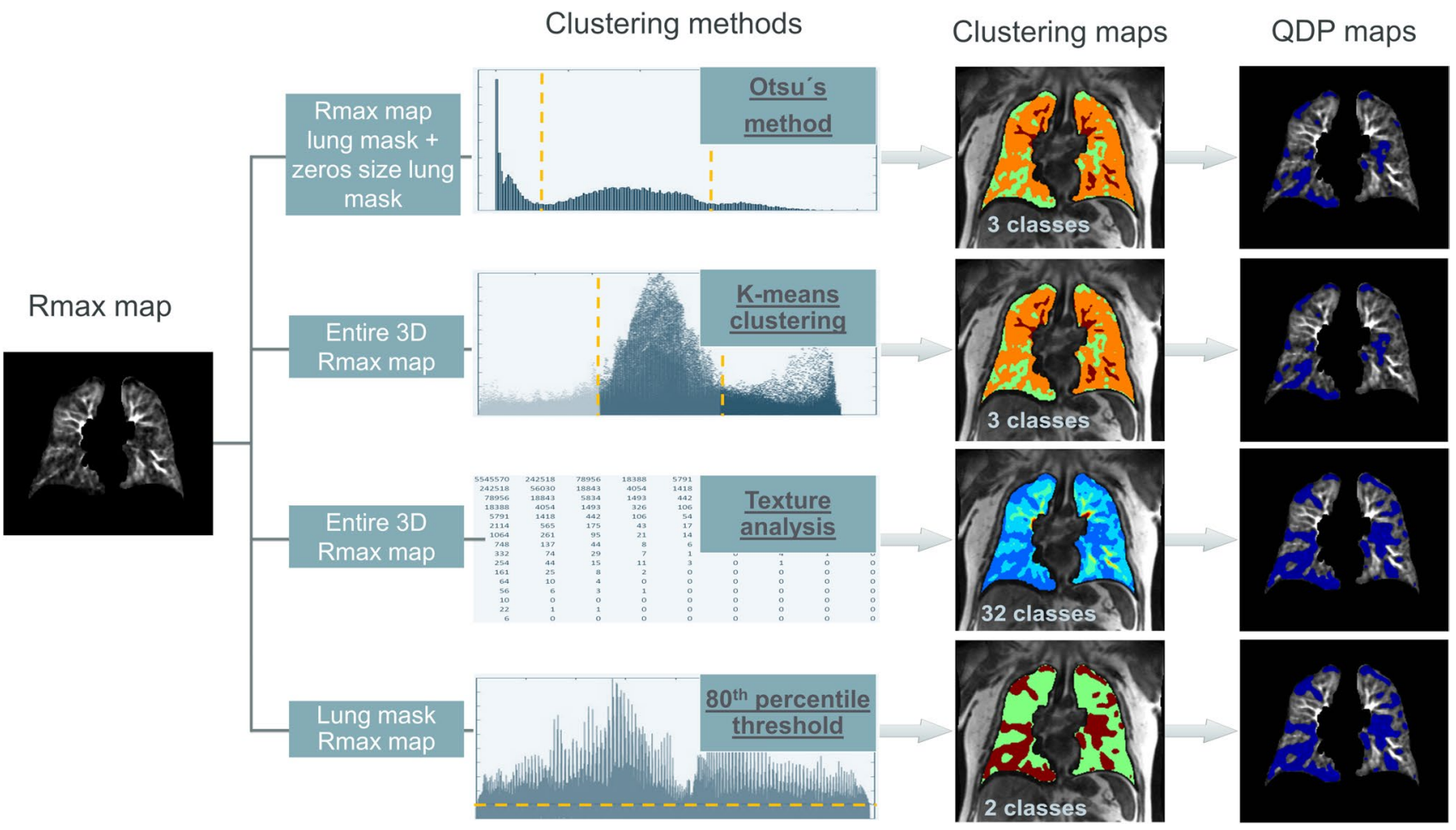

Fig. 1 Flowchart describing the four different approaches to calculate perfusion defects in percent (QDP) using residue function map at the time point of maximum contrast enhancement $\left(R_{\max }\right.$ map). Otsu's method was used to find two thresholds from the histogram containing the intensity values of all voxels within the lung mask of the $R_{\max }$ map and zeros in the same amount as the lung mask's size, resulting in three classes (color map clustering: green $=$ perfusion defects, orange $=$ well perfused, red $=$ vessels). K-means clustering was applied to the entire $R_{\max }$ map to separate the voxels' intensities into three classes (color map clustering: green $=$ perfusion defects, orange $=$ well perfused, red $=$ vessels $)$. The texture analysis utilized

mean \pm standard deviation. Bland-Altman analysis, linear regression, Spearman correlation, Cohen's k, percent agreement, Wilcoxon signed-rank test, Pearson and Filon's z, the co-occurrence matrix of the entire $R_{\max }$ map to separate the voxels into 32 classes (color map clustering: each class with a different color from blue (perfusion defects) to red (vessels)). The $80^{\text {th }}$ percentile of all voxels' intensity inside the lung mask multiplied with 0.5 was used as a threshold between well- and poorly-perfused lung voxels, resulting in 2 classes (color map clustering: red = perfusion defects and green $=$ well perfused). In all approaches, the class with the lowest mean signal intensity from the $R_{\max }$ map was defined as perfusion defect (= QDP). Corresponding QDP maps show resulting perfusion defects in dark blue. Further information about the different QDP calculation approaches can be found in the Supplementary materials

one-way analysis of variance (ANOVA), and scatterplots were used. A $p$ value $<0.05$ was considered statistically significant. Further details are provided in the Supplementary materials.

Table 1 Patient demographics and baseline pulmonary function

\begin{tabular}{|c|c|c|c|c|c|c|c|c|}
\hline & Total & At risk for COPD & Former GOLD 0 & GOLD 1 & GOLD 2 & GOLD 3 & GOLD 4 & $\begin{array}{l}\text { ANOVA } \\
p \text { value }\end{array}$ \\
\hline \multicolumn{9}{|l|}{ Demographics } \\
\hline$n$ & 83 & 5 & 11 & 4 & 29 & 24 & 10 & \\
\hline Age (y) & $65.7 \pm 9.0$ & $65.6 \pm 6.8$ & $69.5 \pm 7.2$ & $62.8 \pm 9.5$ & $66.4 \pm 9.5$ & $64.7 \pm 10.2$ & $62.7 \pm 6.6$ & \\
\hline Sex & $44 \mathrm{f} / 39 \mathrm{~m}$ & $2 \mathrm{f} / 3 \mathrm{~m}$ & $7 \mathrm{f} / 4 \mathrm{~m}$ & $1 \mathrm{f} / 3 \mathrm{~m}$ & $15 \mathrm{f} / 14 \mathrm{~m}$ & $14 \mathrm{f} / 10 \mathrm{~m}$ & $5 \mathrm{f} / 5 \mathrm{~m}$ & \\
\hline Pack years & $36.9 \pm 28.8$ & $32.2 \pm 19.2$ & $12.8 \pm 13.0$ & $23.0 \pm 4.3$ & $34.2 \pm 24.2$ & $39.6 \pm 33.9$ & $74.4 \pm 22.5$ & \\
\hline BMI $\left(\mathrm{kg} / \mathrm{m}^{2}\right)$ & $26.2 \pm 4.6$ & $27.2 \pm 6.5$ & $29.5 \pm 4.2$ & $27.0 \pm 4.9$ & $25.8 \pm 4.5$ & $25.6 \pm 4.7$ & $23.9 \pm 3.1$ & \\
\hline \multicolumn{9}{|l|}{ Pulmonary function } \\
\hline FEV1\%predicted & $55.9 \pm 19.4$ & $81.5 \pm 10.2$ & $78.2 \pm 9.8$ & $85.2 \pm 2.4$ & $61.1 \pm 8.4$ & $41.3 \pm 5.2$ & $26.3 \pm 3.8$ & $<0.001$ \\
\hline FEV1/FVC & $0.56 \pm 0.13$ & $0.74 \pm 0.03$ & $0.76 \pm 0.04$ & $0.64 \pm 0.03$ & $0.58 \pm 0.06$ & $0.47 \pm 0.08$ & $0.37 \pm 0.04$ & $<0.001$ \\
\hline
\end{tabular}

Information about pack-years was available for 45 patients only, BMI data were available for 80 patients only. Data are presented as mean \pm standard deviation. BMI body mass index, COPD chronic obstructive pulmonary disease, FEV1\%predicted forced expiratory volume in $1 \mathrm{~s}$ percent predicted, $F E V 1 / F V C$ ratio between forced expiratory volume in $1 \mathrm{~s}$ and forced vital capacity 


\section{Results}

\section{Patient population and technical feasibility}

The final cohort compromised 83 out of 103 subjects with evaluable baseline DCE-MRI (Table 1). Subjects were excluded before processing the MRI data, with 6 subjects due to missing MRI sequences, 11 subjects due to substantial respiratory artefacts, and 3 subjects due to failed CA application or other substantial artefacts. In 41 subjects, the DCEMRI series were refined by manually removing acquisitions of time points with respiratory artefacts before or after the
Fig. 2 Representative DCE-MRI and CT of a 51 years old female patient with COPD GOLD2 with FEV1\%predicted $=53.26 \%$, $\mathrm{FEV} 1 / \mathrm{FVC}=0.53$, and MRI perfusion score $=10$. a Residue function map at the time point of maximum contrastenhancement ( $R_{\max }$ map), (b) corresponding map of perfusion defects in percent (QDP map, blue) calculated with Otsu's method (QDP $=72.94 \%$ ), (c) corresponding map of the pulmonary blood flow $(\mathrm{PBF}=30.19 \mathrm{ml} / 100 \mathrm{ml} / \mathrm{min})$, (d) corresponding map of the pulmonary blood volume $(\mathrm{PBV}=2.34 \mathrm{ml} / 100 \mathrm{ml}),(\mathbf{e})$ coronal CT and (f) corresponding parametric response map (PRM map) are presented. PRM classifies the voxels of the lung into normal lung tissue $(28.23 \%$, green), functional small airway disease (fSAD $=34.96 \%$, yellow), and emphysema (36.13\%, red)
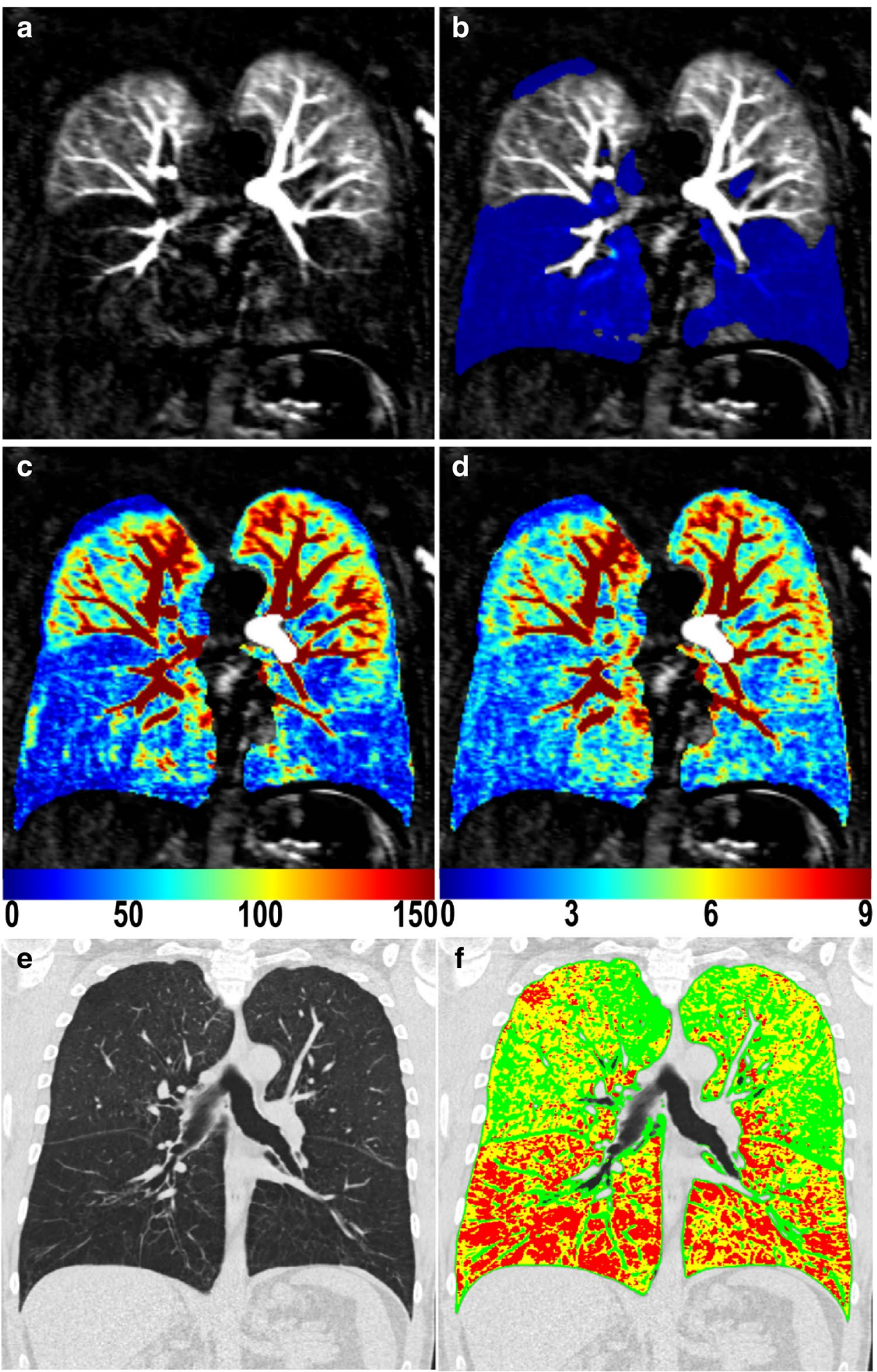
CA bolus passage through the lung, whereby on average 2.6 time points were removed. Lung segmentation masks were generated automatically in all cases. Representative MRI and CT images from a COPD patient with corresponding colorcoded QDP, PBF, PBV, and PRM maps are given in Fig. 2.

\section{Comparison between quantitative perfusion abnormalities and MRI perfusion score}

Mean values for the MRI perfusion parameters per subject group are given in Table 2. All MRI perfusion parameters increased (MRI perfusion score, QDP) or decreased (PBF and PBV) as expected with increasing GOLD severity (GOLD 1-4) (Table 2). The value range of QDP for the whole lung using Otsu's method, k-means clustering, and texture analysis covered approximately $90 \%$ of the maximum theoretical range from 0 to $100 \%$, whereas a smaller value range coverage was observed for the $80^{\text {th }}$ percentile threshold of approximately 53\%. The correlation of QDP with the MRI perfusion score for the whole lung was moderate to strong ( $r=0.68$ to $0.72, p<0.001)$ and the correlations of $\mathrm{PBF}$ and PBV with the MRI perfusion score were moderate ( $r=-0.49$ and $r=-0.54, p<0.001$ ). (Table 3, Fig. 3). Of note,

Table 2 Imaging results per subject group

\begin{tabular}{|c|c|c|c|c|c|c|c|c|}
\hline & Total & At risk for COPD & Former GOLD 0 & GOLD 1 & GOLD 2 & GOLD 3 & GOLD 4 & $\begin{array}{l}\text { ANOVA } \\
p \text { value }\end{array}$ \\
\hline \multicolumn{9}{|l|}{ MRI visual scoring } \\
\hline MRI perfusion score & $9.1 \pm 2.9$ & $8.4 \pm 2.3$ & $7.3 \pm 3.0$ & $7.2 \pm 2.5$ & $9.2 \pm 3.1$ & $9.5 \pm 2.7$ & $11.2 \pm 1.7$ & $<0.01$ \\
\hline \multicolumn{9}{|c|}{ Quantitative DCE-MRI parameters } \\
\hline QDP - Otsu's method & $54.6 \pm 17.8$ & $42.0 \pm 15.0$ & $39.0 \pm 10.3$ & $32.4 \pm 23.5$ & $57.3 \pm 16.2$ & $60.4 \pm 14.4$ & $65.5 \pm 16.8$ & $<0.001$ \\
\hline $\begin{array}{l}\text { QDP - k-means cluster- } \\
\text { ing }\end{array}$ & $52.7 \pm 17.7$ & $40.0 \pm 14.7$ & $36.7 \pm 11.4$ & $30.7 \pm 21.7$ & $55.2 \pm 15.6$ & $58.5 \pm 14.2$ & $64.6 \pm 17.2$ & $<0.001$ \\
\hline QDP - texture analysis & $49.9 \pm 22.6$ & $36.8 \pm 18.2$ & $30.6 \pm 17.8$ & $24.7 \pm 21.1$ & $54.5 \pm 22.2$ & $55.1 \pm 19.0$ & $61.9 \pm 19.9$ & $<0.001$ \\
\hline QDP - $80^{\text {th }}$ percentile & $51.7 \pm 9.3$ & $44.9 \pm 9.1$ & $43.7 \pm 7.4$ & $38.9 \pm 17.2$ & $52.7 \pm 7.6$ & $55.3 \pm 7.1$ & $57.7 \pm 5.3$ & $<0.001$ \\
\hline $\mathrm{PBF}(\mathrm{ml} / 100 \mathrm{ml} / \mathrm{min})$ & $50.6 \pm 24.8$ & $63.9 \pm 27.1$ & $65.9 \pm 26.1$ & $66.9 \pm 23.4$ & $44.5 \pm 21.3$ & $48.5 \pm 27.0$ & $42.8 \pm 18.9$ & $<0.01$ \\
\hline PBV (ml/100 ml) & $3.8 \pm 1.7$ & $4.6 \pm 1.7$ & $5.2 \pm 1.7$ & $5.5 \pm 3.2$ & $3.5 \pm 1.2$ & $3.5 \pm 1.7$ & $3.0 \pm 1.3$ & $<0.001$ \\
\hline \multicolumn{9}{|c|}{$\mathrm{CT}$ parametric response mapping } \\
\hline $\mathrm{PRM}_{\text {Normal }}(\%)$ & $51.4 \pm 21.4$ & $80.1 \pm 9.6$ & $77.1 \pm 21.4$ & $63.2 \pm 12.5$ & $51.2 \pm 17.0$ & $40.2 \pm 11.0$ & $27.0 \pm 7.3$ & $<0.001$ \\
\hline $\mathrm{PRM}_{\text {Emph }}(\%)$ & $13.7 \pm 11.7$ & $5.0 \pm 4.6$ & $5.1 \pm 8.4$ & $5.9 \pm 6.4$ & $12.2 \pm 10.3$ & $16.4 \pm 10.1$ & $31.0 \pm 7.8$ & $<0.001$ \\
\hline $\operatorname{PRM}_{\mathrm{fSAD}}(\%)$ & $34.4 \pm 13.7$ & $14.6 \pm 5.6$ & $17.3 \pm 13.5$ & $30.1 \pm 6.2$ & $35.8 \pm 10.8$ & $42.9 \pm 9.1$ & $41.6 \pm 6.5$ & $<0.001$ \\
\hline $\mathrm{PRM}_{\text {Abnormal }}(\%)$ & $48.6 \pm 21.4$ & $19.9 \pm 9.6$ & $22.9 \pm 21.4$ & $36.8 \pm 12.5$ & $48.8 \pm 17.0$ & $59.8 \pm 11.0$ & $73.0 \pm 7.3$ & $<0.001$ \\
\hline
\end{tabular}

CT parametric response mapping data were only available for 76 patients, Data are presented as mean \pm standard deviation. $C O P D$ chronic obstructive pulmonary disease, Emph emphysema, $f S A D$ functional small airways disease, $P B F$ pulmonary blood flow, $P B V$ pulmonary blood volume, $Q D P$ perfusion defects in percent, $P R M$ parametric response mapping

Table 3 Comparison of quantitative DCE-MRI perfusion parameters with the MRI perfusion score

\begin{tabular}{|c|c|c|c|c|c|c|}
\hline & \multicolumn{4}{|l|}{ QDP } & \multirow{2}{*}{$\begin{array}{l}\mathrm{PBF} \\
(\mathrm{ml} / 100 \mathrm{ml} / \\
\mathrm{min})\end{array}$} & \multirow{2}{*}{$\begin{array}{l}\text { PBV } \\
(\mathrm{ml} / 100 \mathrm{ml})\end{array}$} \\
\hline & Otsu's method & K-means clustering & Texture analysis & $80^{\text {th }}$ percentile & & \\
\hline \multicolumn{7}{|l|}{ MRI perfusion score whole lung } \\
\hline$r$ & $0.72 * * *$ & $0.71 * * *$ & $0.68 * * *$ & $0.67 * * *$ & $-0.49 * * *$ & $-0.54 * * *$ \\
\hline $195 \% \mathrm{CIl}$ & $0.54,0.78$ & $0.55,0.78$ & $0.52,0.77$ & $0.48,0.75$ & $0.27,0.61$ & $0.31,0.64$ \\
\hline Mean diff & $-2.31 \pm 2.13$ & $-2.50 \pm 2.13$ & $-2.76 \pm 2.38$ & $-0.13 \pm 2.25$ & $-5.02 \pm 4.22$ & $-4.52 \pm 4.28$ \\
\hline \multicolumn{7}{|l|}{ MRI perfusion score lobe-based } \\
\hline Cohen’s kappa (k) & 0.48 & 0.47 & 0.46 & 0.39 & & \\
\hline$\%$ Agreement $(\%)$ & 73.29 & 72.69 & 70.28 & 68.67 & & \\
\hline Wilcoxon signed-rank test & 0.06 & 0.27 & $<0.05$ & 0.38 & & \\
\hline
\end{tabular}

For the mean difference analysis in the whole lung, the quantitative values (QDP, PBF, PBV) were normalized to a maximum of 12 for statistical reasons. For comparison by lobe, QDP were converted into discrete values of 0,1 , and 2 at lobe level analogous to the visual scoringsystem (Supplement Table E2) [30]. PBF and PBV could not be transferred to discrete values per lobe. $p$ values smaller than 0.05 in the Wilcoxon signed rank test to evaluate the symmetry of the differences are considered to indicate a non-symmetrical distribution of the differences. $95 \%$ CI 95\% of confidence intervals, Mean diff mean difference \pm standard deviation, $P B F$ pulmonary blood flow, $P B V$ pulmonary blood volume, $Q D P$ perfusion defects in percent, \%Agreement percent agreement. ${ }^{*} p<0.05$, ** $p<0.01$, and $* * * p<0.001$ 
Fig. 3 Association between perfusion defects in percent (QDP), pulmonary blood flow (PBF), and pulmonary blood volume (PBV) with MRI perfusion score. QDP calculated (a) based on Otsu's method showed a range of values between 5.09 and $95.89 \%$, (b) based on k-means clustering between 4.91 and $95.23 \%$, (c) based on texture analysis between 1.28 and $93.73 \%$, and (d) based on the $80^{\text {th }}$ percentile threshold between 15.17 and $68.82 \%$, the latter being compression of the observed value range compared to the other QDP quantification methods. e PBF showed a range of observed values between 7.30 and $147.03 \mathrm{ml} / \mathrm{min} / 100 \mathrm{ml}$ and (f) PBV between 1.37 and $9.96 \mathrm{ml} / 100 \mathrm{ml}$. Respective linear regression lines, Spearman correlation coefficients, and corresponding $p$ values are given in the plots
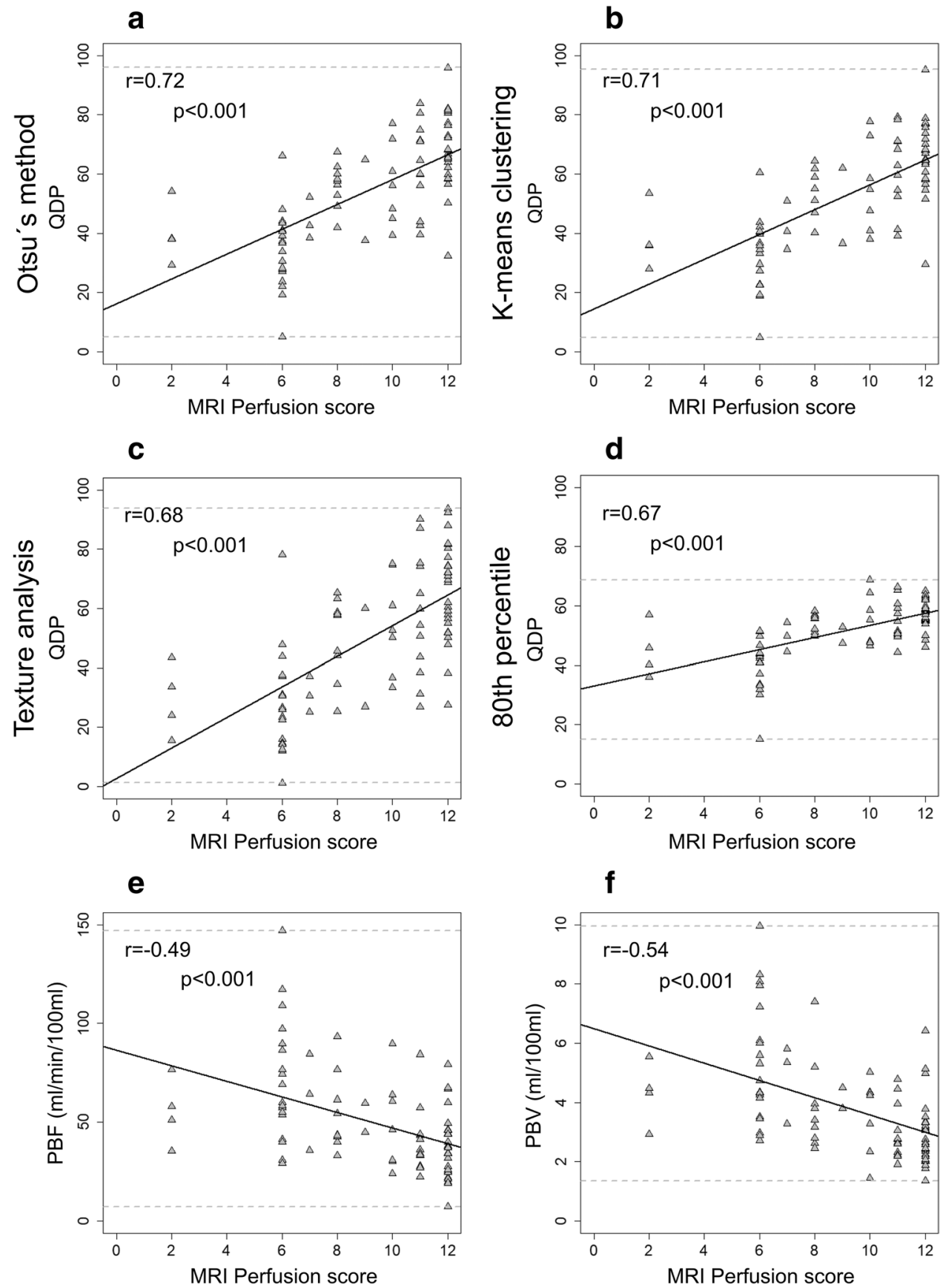

QDP correlated significantly higher (Pearson and Filon's z) with the MRI perfusion score than PBF and PBV $(p<0.001-0.05)$.

In a lobe-based comparison between QDP, using converted discrete values, and MRI perfusion score Cohen's $\mathrm{k}$ revealed moderate agreements, ranging between 0.39 for the $80^{\text {th }}$ percentile threshold and 0.48 for Otsu's method. A bias in the symmetrical distribution in the differences between QDP and MRI perfusion score was evaluated with the Wilcoxon signed-rank test. A bias was observed for QDP quantified with texture analysis (Table 3).

\section{Comparison between MRI perfusion and CT parametric response mapping}

CT PRM abnormalities increased with increasing GOLD severity (GOLD 1-4) (Table 2). All QDP quantification methods correlated strongly with $\mathrm{PRM}_{\text {Emph }}(r=0.75$ to 0.70 , $p<0.001)$ and moderately with $\mathrm{PRM}_{\text {Abnormal }}(r=0.65$ to $0.61, p<0.001)$, but only weakly with $\operatorname{PRM}_{\mathrm{fSAD}}(r=0.34$ to $0.37, p<0.01)$. In comparison, PBF and PBV were moderately correlated with $\mathrm{PRM}_{\mathrm{Emph}}(r=-0.51$ and $r=-0.64$, 
Table 4 Comparison of DCE-MRI perfusion parameters with CT parametric response mapping and pulmonary function parameters

\begin{tabular}{|c|c|c|c|c|c|c|c|}
\hline & \multirow{2}{*}{$\begin{array}{l}\text { MRI perfusion } \\
\text { score }\end{array}$} & \multicolumn{4}{|l|}{ QDP } & \multirow{2}{*}{$\begin{array}{l}\text { PBF } \\
(\mathrm{ml} / 100 \mathrm{ml} / \mathrm{min})\end{array}$} & \multirow{2}{*}{$\begin{array}{l}\text { PBV } \\
(\mathrm{ml} / 100 \mathrm{ml}\end{array}$} \\
\hline & & Otsu's method & K-means clustering & Texture analysis & 80th percentile & & \\
\hline \multicolumn{8}{|c|}{$\operatorname{PRM}_{\text {Abnormal }}(\%)$} \\
\hline$r$ & $0.50 * * *$ & $0.63 * * *$ & $0.65^{* * *}$ & $0.62 * * *$ & $0.61 * * *$ & $-0.51 * * *$ & $-0.63^{* * *}$ \\
\hline $195 \% \mathrm{CI} \mid$ & $0.30,0.65$ & $0.48,0.76$ & $0.49,0.78$ & $0.46,0.75$ & $0.42,0.74$ & $0.32,0.66$ & $0.48,0.75$ \\
\hline Mean diff & - & $6.03 \pm 16.94$ & $4.15 \pm 16.39$ & $1.47 \pm 19.30$ & $3.23 \pm 17.08$ & - & - \\
\hline \multicolumn{8}{|l|}{$\mathrm{PRM}_{\text {Emph }}(\%)$} \\
\hline$R$ & $0.56^{* * *}$ & $0.74 * * *$ & $0.75 * * *$ & $0.70 * * *$ & $0.70 * * *$ & $-0.51 * * *$ & $-0.64 * * *$ \\
\hline $195 \% \mathrm{CI}$ & $0.38,0.69$ & $0.62,0.83$ & $0.62,0.84$ & $0.55,0.81$ & $0.56,0.80$ & $0.32,0.66$ & $0.50,0.77$ \\
\hline Mean diff & - & $40.4 \pm 13.28$ & $38.53 \pm 12.91$ & $35.85 \pm 17.85$ & $37.61 \pm 9.17$ & - & - \\
\hline \multicolumn{8}{|l|}{$\operatorname{PRM}_{\mathrm{fSAD}}(\%)$} \\
\hline$r$ & $0.29 * *$ & $0.35^{* *}$ & $0.37 * *$ & $0.37 * *$ & $0.34 * *$ & $-0.38 * * *$ & $-0.40 * * *$ \\
\hline $195 \% \mathrm{CI}$ & $0.07,0.46$ & $0.12,0.55$ & $0.15,0.55$ & $0.14,0.55$ & $0.11,0.55$ & $0.16,0.55$ & $0.19,0.58$ \\
\hline Mean diff & - & $19.68 \pm 17.67$ & $17.81 \pm 17.28$ & $15.12 \pm 21.56$ & $16.88 \pm 12.79$ & - & - \\
\hline \multicolumn{8}{|c|}{ FEV1\%predicted } \\
\hline$r$ & $-0.39 * * *$ & $-0.43 * * *$ & $-0.44 * * *$ & $-0.37 * * *$ & $-0.49 * * *$ & $0.26^{*}$ & $0.38 * * *$ \\
\hline $195 \% \mathrm{CI}$ & $0.18,0.56$ & $0.22,0.59$ & $0.25,0.61$ & $0.15,0.54$ & $0.32,0.64$ & $0.04,0.45$ & $0.19,0.54$ \\
\hline \multicolumn{8}{|l|}{ FEV1/FVC } \\
\hline$r$ & $-0.45^{* * *}$ & $-0.50 * * *$ & $-0.51 * * *$ & $-0.41 * * *$ & $-0.54 * * *$ & $0.28 * *$ & $0.40 * * *$ \\
\hline $195 \% \mathrm{CI}$ & $0.26,0.62$ & $0.31,0.65$ & $0.32,0.65$ & $0.21,0.58$ & $0.37,0.68$ & $0.07,0.47$ & $0.20,0.57$ \\
\hline
\end{tabular}

Only for 76 patients CT parametric response mapping data were available. Emph emphysema, FEV1\%predicted forced expiratory volume in $1 \mathrm{~s}$ percent predicted, $F E V I / F V C$ ratio between forced expiratory volume in $1 \mathrm{~s}$ and forced vital capacity, $f S A D$ functional small airways disease, Mean diff mean difference \pm standard deviation, $P B F$ pulmonary blood flow, $P B V$ pulmonary blood volume, $Q D P$ perfusion defects in percent, PFT pulmonary function testing, $P R M$ parametric response mapping. $* p<0.05, * * p<0.01$, and $* * * p<0.001$

$p<0.001$, respectively) and $\mathrm{PRM}_{\text {Abnormal }}(r=-0.51$ and $r=-0.63, p<0.001$, respectively), but weakly with $\operatorname{PRM}_{\mathrm{fSAD}}(r=-0.38$ and $r=-0.40, p<0.001$, respectively) (Table 4). Of note, QDP based on Otsu's method and k-means clustering correlated significantly higher (Pearson

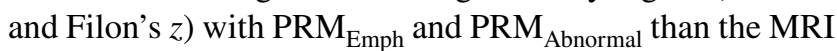
perfusion score, $\mathrm{PBF}$, and PBV.

In Bland-Altman analyses, QDP was distinctly higher than $\mathrm{PRM}_{\text {Emphysema }}$ (mean difference QDP$\mathrm{PRM}_{\text {Emph }}=35.85 \pm 17.85$ to $\left.40.4 \pm 13.28\right)$ and $\mathrm{PRM}_{\mathrm{fSAD}}$ (mean difference QDP-PRM $\mathrm{PSAD}_{\mathrm{fAD}}=15.12 \pm 21.56$ to $19.68 \pm 17.67)$. When considering emphysema and fSAD together (PRM $\mathrm{Pbnormal})$, the mean difference was reduced and close to zero (mean difference QDP-PRMAbnormal $=1.47 \pm 19.30$ to $6.03 \pm 16.94$ ) (Table 4, Fig. 4, and Supplementary Fig. 2).

\section{Comparison between MRI perfusion and pulmonary function testing}

QDP and the MRI perfusion score correlated moderately with FEV1/FVC $(r=-0.41$ to $-0.54, p<0.001)$. Only a weak correlation of $r=0.28(p<0.05)$ and a moderate correlation of $r=0.40(p<0.001)$ were found for PBF and PBV, respectively (Table 4).
No statistically significant differences were observed between the correlation coefficients of QDP and the MRI perfusion score with PFT. However, QDP correlated significantly higher with FEV1/FVC than PBF (except for QDP based on $80^{\text {th }}$ percentile). QDP based on k-means clustering correlated significantly higher with FEV1/FVC than PBV.

\section{Discussion}

We developed new methods to quantify QDP using DCEMRI, which combine the advantages of unsupervised image clustering algorithms and mathematical models of tracer kinetics. Our results showed that (a) QDP based on Otsu's method showed the highest consistency with the MRI perfusion score and (b) QDP is clinically meaningful due to its significant associations with the MRI perfusion score, CT PRM indices of emphysema and fSAD, and PFT. Furthermore, assessing pulmonary perfusion abnormalities as "defect-percent" showed advantages over the assessment of $\mathrm{PBF}$ and PBV, as we observed higher correlations with CT PRM indices and PFT for QDP.

The quantification of the conventional perfusion metrics $\mathrm{PBF}$ and PBV is based on $\mathrm{R}(\mathrm{t})$ map of the lung, which is often subject to high variability due to limited temporal 

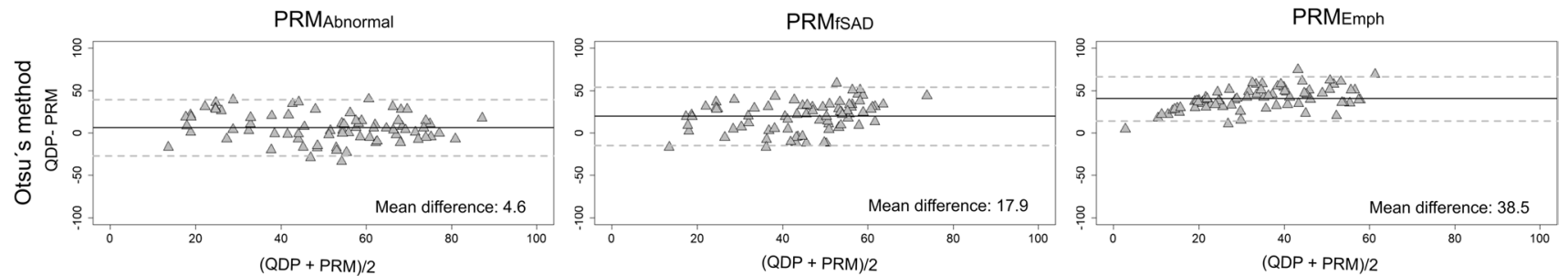

Fig. 4 Bland-Altman plot between perfusion defects in percent (QDP) based on Otsu's method using DCE-MRI and CT parametric response mapping (PRM) indices. Solid lines represent mean differences and dashed lines represent limits of agreements (+ -1.96SD) between QDP calculated with Otsu's method and the PRM indices abnormal lung $\left(\mathrm{PRM}_{\mathrm{Abnormal}}\right)$, functional small airways disease

resolution [35], non-linearity of the CA concentration to signal relationship [6, 7], overall low contrast-to-noise-ratio in the lung parenchyma, and respiratory motion artefacts. Consequently, this leads to physiologically undesirable and unreliable values in the $\mathrm{R}(\mathrm{t})$ map, which, in turn, increases the variability of PBF and PBV [8]. QDP uses the same $\mathrm{R}(\mathrm{t})$ map as a basis for the calculation and thus exploits the advantages of mathematical models based on the principles of tracer kinetics for non-diffusible tracers. However, QDP does not use the individual voxel values such as PBF and PBV as the next step, but instead uses unsupervised image clustering algorithms to identify poorly-perfused voxels. Hence, the influence of certain challenges of DCE-MRI sequences in the lungs is reduced, and thus, the calculation should be more robust. Overall, we observed higher correlations of QDP with the MRI perfusion score, CT PRM indices, and PFT compared to PBF and PBV.

Since QDP is determined using an automated computer algorithm, the calculation is time-efficient and allows for a more detailed assessment of perfusion abnormalities compared to visual MRI perfusion scoring. Furthermore, it is user-independent and eliminates potential intra- and interreader variabilities $[2,19]$. In this study, QDP was comparable to the MRI perfusion score and showed even higher correlations with CT PRM indices and PFT.

We compared four different QDP quantification approaches to assess the strengths and weaknesses of each approach. We not only mainly developed and optimized QDP to reflect the MRI perfusion score, but also compared the different QDP methods for method development purposes with CT PRM and PFT parameters, since perfusion abnormalities are associated with both airflow limitations and the destruction of lung parenchyma. We propose to use QDP based on Otsu's method for future clinical studies, because it showed overall the highest level of agreement with the MRI perfusion score and high correlations with CT PRM and PFT parameters. However, it must be mentioned that all presented clustering approaches require a predefined
$\left(\mathrm{PRM}_{\mathrm{fSAD}}\right)$, and emphysema $\left(\mathrm{PRM}_{\mathrm{Emph}}\right)$. Please note that the mean difference between QDP and $\mathrm{PRM}_{\text {Abnormal }}$ is close to zero. For the other three QDP calculation approaches, i.e., k-means clustering, texture analysis, and $80^{\text {th }}$ percentile threshold, the Bland-Altman plots are depicted in Supplementary Fig. 2

number of classes, which implies that the methods must find perfusion defects and well-perfused tissue. Consequently, clustering approaches may potentially overestimate mild disease and underestimate very severe disease. Minor perfusion defects under 7.5\% should be rejected as no perfusion defects, similar to what was done in the lobe-based comparison [30]. In previous studies, percentile thresholds were used to quantify pulmonary perfusion defects using DCE-MRI $[12,36]$. We noticed a compression of the range of observed values for the percentile method compared to the other methods, which is caused by its underlying calculation method, regardless of the used percentile and factor. This compressed value range affects the comparability with the MRI perfusion score. The percentile and factor used here were empirically determined in a previous study [36] and may not be transferable to data of other MRI acquisition techniques or scanner types. The use of texture analysis or other more advanced analysis methods might be challenging due to the low contrast-to-noise ratio and pronounced artefacts in DCE-MRI of the lungs.

The general concept of describing functional lung abnormalities as "defect-percent" appears to be clinically meaningful as ventilation defects in percent quantified from hyperpolarized gas MRI have already been used to monitor treatment response in $\mathrm{CF}[37,38]$. Initial studies on the quantification of pulmonary perfusion as "defect-percent" using DCE-MRI and FD-MRI also indicate its potential, but further evaluation was missing to date $[12,30]$. The quantification of perfusion defects may provide complementary or overlapping information to ventilation defects, because pulmonary perfusion and ventilation are related through the HPV. However, studies are indicating that the ventilation-perfusion match in COPD deteriorates with an increasing level of emphysema [39]. Here the ability to block the HPV in inflamed lung regions seems to be suspended in emphysema-susceptible patients and may therefore contribute to emphysema development [40]. In addition, studies in COPD could already demonstrate that fSAD precedes the development of emphysema using CT [41, 42]. 
Consequently, the comparison of perfusion abnormalities with $\mathrm{PRM}_{\mathrm{fSAD}}$ can confirm the hypothesis that perfusion abnormalities may serve as a prognostic biomarker for emphysema progression in COPD. Moreover, it was speculated whether impaired pulmonary perfusion is a reversible component of the COPD pathogenesis [1], which was already demonstrated in patients with CF [20] and COPD [43]. In a previous study, no association between PBF using DCE-MRI and small airways disease using CT was observed [1]. We observed significant correlations between QDP and the PRM parameters $\mathrm{PRM}_{\mathrm{Emph}}, \mathrm{PRM}_{\mathrm{fSAD}}$, and $\mathrm{PRM}_{\mathrm{Abnormal}}$. In addition, the extent of perfusions defects from MRI corresponds to the CT-derived extent of abnormal lung (emphysema and fSAD). Considered separately, more perfusion defects were observed than emphysema or fSAD. This indicates that QDP represents the entire parenchymal abnormalities detected by PRM.

We found moderate correlations between QDP and FEV1\%predicted or FEV1/FVC, indicating a relationship between perfusion abnormalities and airflow limitation. This is consistent with previous studies $[12,44]$ and in agreement with the understanding that a reduction in FEV1\%predicted is mainly driven by large airway obstruction, whereas pulmonary perfusion abnormalities probably reflect also small airway pathologies $[1,20]$.

One technical limitation of this study is the use of data from one single scanner. Other QDP quantification methods, or PBF and PBV, could be superior in future studies with different patient populations and/or other DCE-MRI sequences with better image quality. The DCE-MRI quality issues in the lung are primarily caused by the measurement technique to achieve the necessary temporal resolution, together with the low proton density and pronounced susceptibility artefacts in the lungs. These issues even get worse with disease severity in COPD. Furthermore, the long acquisition time in inspiratory breath-hold is challenging for many COPD patients. The acquisition time is pre-defined as individual circulation times of the CA through the lungs are unknown. However, only the time points of the first CA-bolus passage through the lungs are required for the perfusion quantification. The removal of time points affected by respiratory motion could influence the PBF and PBV quantification (absolute flow and volume values), but distinctly less the QDP calculation (relative values due to the intrinsic normalization of the clustering algorithms). Attempts to correct respiratory motion with image registration have not succeeded so far. Since cross-sectional correlations do not prove causality, the relevance of perfusion abnormalities for the development of emphysema must be further investigated in large longitudinal studies, ideally with a regional comparison between quantitative CT and quantitative MRI. In addition, interventional studies are prerequisites before QDP can be used in clinical drug development and the robustness and repeatability of the quantitative perfusion parameters must be assessed in further method validation studies.

This study demonstrates QDP quantified using DCE-MRI is associated with established markers of disease severity in COPD. It corresponds to the extent of emphysema and fSAD in percent quantified using $\mathrm{CT}$, indicating that pulmonary perfusion abnormalities themselves may contribute to or at least precede the development of irreversible emphysema. QDP showed considerable advantages over PBF and PBV, which were used in previous COPD studies. We conclude that QDP based on Otsu's method from DCE-MRI is a promising novel biomarker for clinical trials in COPD.

Supplementary Information The online version contains supplementary material available at https://doi.org/10.1007/s00330-021-08229-6.

Acknowledgements We thank all patients for their participation in this study. We also thank the MRI technicians at the Thoraxklinik at the University of Heidelberg for their technical assistance in performing the MRI examinations.

Funding Open Access funding enabled and organized by Projekt DEAL. This study has received funding from Bayer Vital GmbH (sponsored the MRI contrast agent material).

\section{Declarations}

Guarantor The scientific guarantor of this publication is Prof. Dr. med. Mark O. Wielpütz, EDIR, MHBA.

Conflict of interest The authors of this manuscript declare relationships with the following companies: Marilisa Schiwek and Frank Risse are employees of Boehringer Ingelheim.

The (remaining) authors of this manuscript declare no relationships with any companies whose products or services may be related to the subject matter of the article.

Statistics and biometry Carina Ittrich kindly provided statistical advice for this manuscript.

Informed consent Written informed consent was obtained from all subjects in this study.

Ethical approval Institutional Review Board approval was obtained.

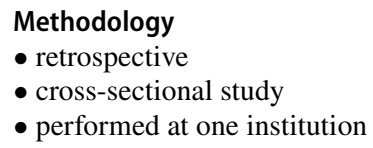

Open Access This article is licensed under a Creative Commons Attribution 4.0 International License, which permits use, sharing, adaptation, distribution and reproduction in any medium or format, as long as you give appropriate credit to the original author(s) and the source, provide a link to the Creative Commons licence, and indicate if changes were made. The images or other third party material in this article are included in the article's Creative Commons licence, unless indicated otherwise in a credit line to the material. If material is not included in the article's Creative Commons licence and your intended use is not permitted by statutory regulation or exceeds the permitted use, you will 
need to obtain permission directly from the copyright holder. To view a copy of this licence, visit http://creativecommons.org/licenses/by/4.0/.

\section{References}

1. Hueper K, Vogel-Claussen J, Parikh MA et al (2015) Pulmonary microvascular blood flow in mild chronic obstructive pulmonary disease and emphysema. The MESA COPD Study. Am J Respir Crit Care Med 192:570-580. https://doi.org/10.1164/rccm.201411-2120oc

2. Eichinger M, Optazaite DE, Kopp-Schneider A et al (2012) Morphologic and functional scoring of cystic fibrosis lung disease using MRI. Eur J Radiol 81:1321-1329. https://doi.org/10.1016/j.ejrad.2011.02.045

3. Wielpütz MO, Eichinger M, Wege S et al (2019) Midterm reproducibility of chest magnetic resonance imaging in adults with clinically stable cystic fibrosis and chronic obstructive pulmonary disease. Am J Resp Crit Care 200:103-107. https://doi.org/10. 1164/rccm.201812-2356le

4. Ohno Y, Hatabu H, Murase K et al (2004) Quantitative assessment of regional pulmonary perfusion in the entire lung using three-dimensional ultrafast dynamic contrast-enhanced magnetic resonance imaging: preliminary experience in 40 subjects. J Magn Reson Imaging 20:353-365. https://doi.org/10.1002/jmri.20137

5. Hatabu H, Tadamura E, Levin DL et al (1999) Quantitative assessment of pulmonary perfusion with dynamic contrast-enhanced MRI. Magnet Reson Med 42:1033-1038. https://doi.org/10.1002/ (sici)1522-2594(199912)42:6\%3c1033::aid-mrm7\%3e3.0.co;2-7

6. Puderbach M, Risse F, Biederer J et al (2008) In vivo Gd-DTPA concentration for MR lung perfusion measurements: assessment with computed tomography in a porcine model. Eur Radiol 18:2102-2107. https://doi.org/10.1007/s00330-008-0974-1

7. Neeb D, Kunz RP, Ley S et al (2009) Quantification of pulmonary blood flow (PBF): Validation of perfusion MRI and nonlinear contrast agent (CA) dose correction with $\mathrm{H} 215 \mathrm{O}$ positron emission tomography (PET). Magnet Reson Med 62:476-487. https://doi. org/10.1002/mrm.22025

8. Ley-Zaporozhan J, Molinari F, Risse F et al (2011) Repeatability and reproducibility of quantitative whole-lung perfusion magnetic resonance imaging. J Thorac Imaging 26:230-239. https://doi.org/ 10.1097/rti.0b013e3181e48c36

9. Ter-Karapetyan A, Triphan SMF, Jobst BJ et al (2018) Towards quantitative perfusion MRI of the lung in COPD: the problem of short-term repeatability. PLoS One 13:e0208587. https://doi.org/ 10.1371/journal.pone.0208587

10. Woodhouse N, Wild JM, Paley MNJ et al (2005) Combined helium-3/proton magnetic resonance imaging measurement of ventilated lung volumes in smokers compared to never-smokers. J Magn Reson Imaging 21:365-369. https://doi.org/10.1002/jmri.20290

11. Fain SB, Gonzalez-Fernandez G, Peterson ET et al (2008) Evaluation of structure-function relationships in asthma using multidetector CT and hyperpolarized He-3 MRI. Acad Radiol 15:753762. https://doi.org/10.1016/j.acra.2007.10.019

12. Kaireit TF, Voskrebenzev A, Gutberlet M et al (2019) Comparison of quantitative regional perfusion-weighted phase resolved functional lung (PREFUL) MRI with dynamic gadolinium-enhanced regional pulmonary perfusion MRI in COPD patients. J Magn Reson Imaging 49:1122-1132. https://doi.org/10.1002/jmri.26342

13. Eddy RL, Parraga G (2020) Pulmonary xenon-129 MRI: new opportunities to unravel enigmas in respiratory medicine. Eur Respir J 55:1901987. https://doi.org/10.1183/13993003.01987-2019

14. Capaldi DPI, Eddy RL, Svenningsen S et al (2018) Free-breathing pulmonary MR imaging to quantify regional ventilation. Radiology 287:693-704. https://doi.org/10.1148/radiol.2018171993

15. Mathew L, Kirby M, Etemad-Rezai R et al (2011) Hyperpolarized $3 \mathrm{He}$ magnetic resonance imaging: preliminary evaluation of phenotyping potential in chronic obstructive pulmonary disease. Eur J Radiol 79:140-146. https://doi.org/10.1016/j.ejrad.2009.10. 028

16. Couch MJ, Thomen R, Kanhere N et al (2019) A two-center analysis of hyperpolarized 129Xe lung MRI in stable pediatric cystic fibrosis: potential as a biomarker for multi-site trials. J Cyst Fibros 18:728-733. https://doi.org/10.1016/j.jcf.2019.03.005

17. Jörres R, Welte T, Bals R et al (2010) Einfluss systemischer Manifestationen und Komorbiditäten auf den klinischen Zustand und den Verlauf bei COPD. Dtsch Med Wochenschr 135:446-449. https://doi.org/10.1055/s-0030-1249185

18. Singh D, Agusti A, Anzueto A et al (2019) Global strategy for the diagnosis, management, and prevention of chronic obstructive lung disease: the GOLD science committee report 2019. Eur Respir J 53:1900164. https://doi.org/10.1183/13993003. 00164-2019

19. Jobst BJ, Triphan SMF, Sedlaczek O et al (2015) Functional lung MRI in chronic obstructive pulmonary disease: comparison of T1 mapping, oxygen-enhanced T1 mapping and dynamic contrast enhanced perfusion. PLoS One 10:e121520. https://doi.org/10. 1371/journal.pone.0121520

20. Wielpütz MO, Puderbach M, Kopp-Schneider A et al (2014) Magnetic resonance imaging detects changes in structure and perfusion, and response to therapy in early cystic fibrosis lung disease. Am J Respir Crit Care Med 189:956-965. https://doi.org/10.1164/ rccm.201309-1659oc

21. Triphan SMF, Biederer J, Burmester K et al (2018) Design and application of an MR reference phantom for multicentre lung imaging trials. PLoS One 13:e0199148. https://doi.org/10.1371/ journal.pone.0199148

22. Korosec FR, Frayne R, Grist TM, Mistretta CA (1996) Timeresolved contrast-enhanced 3D MR angiography. Magnet Reson Med 36:345-351. https://doi.org/10.1002/mrm.1910360304

23. Eichinger M, Puderbach M, Fink C et al (2006) Contrast-enhanced 3D MRI of lung perfusion in children with cystic fibrosis-initial results. Eur Radiol 16:2147-2152. https://doi.org/10.1007/ s00330-006-0257-7

24. Sileo C, Corvol H, Boelle P-Y et al (2014) HRCT and MRI of the lung in children with cystic fibrosis: comparison of different scoring systems. J Cyst Fibros 13:198-204. https://doi.org/10.1016/j. jcf.2013.09.003

25. Kohlmann P, Laue H, Krass S, Peitgen H-O (2011) Fully-automatic determination of the arterial input function for dynamic contrast-enhanced pulmonary MR imaging. Proceedings of Medical Image Understanding and Analysis 281-285. http://www. bmva.org/miua/2011/miua-11-43.pdf. Accessed 1 Sep 2020

26. Sourbron S, Dujardin M, Makkat S, Luypaert R (2006) Pixelby-pixel deconvolution of bolus-tracking data: optimization and implementation. Phys Med Biol 52:429-447. https://doi.org/10. 1088/0031-9155/52/2/009

27. Zierler KL (1962) Theoretical basis of indicator-dilution methods for measuring flow and volume. Circ Res 10:393-407. https://doi. org/10.1161/01.res.10.3.393

28. Meier P, Zierler KL (1954) On the theory of the indicator-dilution method for measurement of blood flow and volume. J Appl Physiol 6:731-744. https://doi.org/10.1152/jappl.1954.6.12.731

29. Kohlmann P, Strehlow J, Jobst B et al (2015) Automatic lung segmentation method for MRI-based lung perfusion studies of patients with chronic obstructive pulmonary disease. Int J Comput Assist Radiol Surg 10:403-417. https://doi.org/10.1007/ s11548-014-1090-0

30. Bauman G, Puderbach M, Heimann T et al (2013) Validation of Fourier decomposition MRI with dynamic contrast-enhanced MRI using visual and automated scoring of pulmonary perfusion in young cystic fibrosis patients. Eur J Radiol 82:2371-2377. https:// doi.org/10.1016/j.ejrad.2013.08.018 
31. Konietzke P, Wielpütz MO, Wagner WL et al (2020) Quantitative CT detects progression in COPD patients with severe emphysema in a 3-month interval. Eur Radiol 30:2502-2512. https://doi.org/ 10.1007/s00330-019-06577-y

32. Jobst BJ, Weinheimer O, Trauth $\mathrm{M}$ et al (2018) Effect of smoking cessation on quantitative computed tomography in smokers at risk in a lung cancer screening population. Eur Radiol 28:807-815. https://doi.org/10.1007/s00330-017-5030-6

33. Galbán CJ, Han MK, Boes JL et al (2012) Computed tomographybased biomarker provides unique signature for diagnosis of COPD phenotypes and disease progression. Nat Med 18:1711-1715. https://doi.org/10.1038/nm.2971

34. Graham BL, Steenbruggen I, Miller MR et al (2019) Standardization of spirometry 2019 update. An Official American Thoracic Society and European Respiratory Society Technical Statement. Am J Resp Crit Care 200:e70-e88. https://doi.org/10.1164/rccm. 201908-1590st

35. Ingrisch M, Dietrich O, Attenberger UI et al (2010) Quantitative pulmonary perfusion magnetic resonance imaging. Invest Radiol 45:7-14. https://doi.org/10.1097/rli.0b013e3181bc2d0c

36. Heimann T, Eichinger M, Bauman G et al (2012) Automated scoring of regional lung perfusion in children from contrast enhanced 3D MRI. Proc SPIE 8315, Medical Imaging 2012: ComputerAided Diagnosis, 83150U. https://doi.org/10.1117/12.911946

37. Altes TA, Johnson M, Fidler M et al (2017) Use of hyperpolarized helium-3 MRI to assess response to ivacaftor treatment in patients with cystic fibrosis. J Cyst Fibros 16:267-274. https://doi.org/10. 1016/j.jcf.2016.12.004

38. Rayment JH, Couch MJ, McDonald N et al (2019) Hyperpolarised 129Xe magnetic resonance imaging to monitor treatment response in children with cystic fibrosis. Eur Respir J 53:1802188. https:// doi.org/10.1183/13993003.02188-2018

39. Barbera JA, Ramirez J, Roca J et al (1990) Lung structure and gas exchange in mild chronic obstructive pulmonary disease. Am Rev
Respir Dis 141:895-901. https://doi.org/10.1164/ajrccm/141.4_ pt_1.895

40. Alford SK, van Beek EJ, McLennan G, Hoffman EA (2010) Heterogeneity of pulmonary perfusion as a mechanistic image-based phenotype in emphysema susceptible smokers. Proc Natl Acad Sci USA 107:7485-7490. https://doi.org/10.1073/pnas.0913880107

41. Labaki WW, Gu T, Murray S et al (2019) Voxel-wise longitudinal parametric response mapping analysis of chest computed tomography in smokers. Acad Radiol 26:306-312. https://doi.org/10. 1016/j.acra.2019.02.003

42. Boes JL, Hoff BA, Bule M et al (2015) Parametric response mapping monitors temporal changes on lung CT scans in the Subpopulations and Intermediate Outcome Measures in COPD Study (SPIROMICS). Acad Radiol 22:186-194. https://doi.org/ 10.1016/j.acra.2014.08.015

43. Vogel-Claussen J, Schönfeld C-O, Kaireit TF et al (2019) Effect of indacaterol/glycopyrronium on pulmonary perfusion and ventilation in hyperinflated patients with chronic obstructive pulmonary disease (CLAIM). A double-blind, randomized, crossover trial. Am J Resp Crit Care 199:1086-1096. https://doi.org/10.1164/ rccm.201805-0995oc

44. Xia Y, Guan Y, Fan L et al (2014) Dynamic contrast enhanced magnetic resonance perfusion imaging in high-risk smokers and smoking-related COPD: correlations with pulmonary function tests and quantitative computed tomography. COPD 11:510-520. https://doi.org/10.3109/15412555.2014.948990

Publisher's note Springer Nature remains neutral with regard to jurisdictional claims in published maps and institutional affiliations. 